\title{
Safety issues of capacitor banks in medium voltage systems
}

\begin{abstract}
This paper describes and illustrates the important factors concerning the safety issues of capacitor banks in medium voltage (MV) power system networks. It also explains the protective issues that should be considered. A detailed account on failures and risks of capacitors during the switching operations in power distribution systems is presented. Procedures of safety, techniques of protection, existing solutions to avoid equipment damage, as well as maintaining system safety and reliability have been discussed.
\end{abstract}

Keyword: Capacitor; Medium voltage network; Protection 